\title{
Decreased bacterial growth on titanium nanoscale topographies created by ion beam assisted evaporation
}

This article was published in the following Dove Press journal:

International Journal of Nanomedicine

9 February 2017

Number of times this article has been viewed

\author{
Michelle Stolzoff' \\ Jason E Burns ${ }^{2}$ \\ Arash Aslani \\ Eric J Tobin ${ }^{2}$ \\ Congtin Nguyen' \\ Nicholas De La Torre ${ }^{3}$ \\ Negar H Golshan ${ }^{3}$ \\ Katherine S Ziemer ${ }^{3}$ \\ Thomas J Webster ${ }^{1,3,4}$ \\ 'Department of Bioengineering, \\ Northeastern University, Boston, \\ ${ }^{2} \mathrm{~N} 2$ Biomedical, Bedford, MA, \\ ${ }^{3}$ Department of Chemical Engineering, \\ Northeastern University, Boston, \\ MA, USA $;{ }^{4}$ Center of Excellence \\ for Advanced Materials Research, \\ University of King Abdulaziz, Jeddah, \\ Saudi Arabia
}

\begin{abstract}
Titanium is one of the most widely used materials for orthopedic implants, yet it has exhibited significant complications in the short and long term, largely resulting from poor cell-material interactions. Among these many modes of failure, bacterial infection at the site of implantation has become a greater concern with the rise of antibiotic-resistant bacteria. Nanostructured surfaces have been found to prevent bacterial colonization on many surfaces, including nanotextured titanium. In many cases, specific nanoscale roughness values and resulting surface energies have been considered to be "bactericidal"; here, we explore the use of ion beam evaporation as a novel technique to create nanoscale topographical features that can reduce bacterial density. Specifically, we investigated the relationship between the roughness and titanium nanofeature shapes and sizes, in which smaller, more regularly spaced nanofeatures (specifically $40-50 \mathrm{~nm}$ tall peaks spaced $\sim 0.25 \mu \mathrm{m}$ apart) were found to have more effect than surfaces with high roughness values alone.
\end{abstract}

Keywords: titanium, nanostructures, bacteria, bone ingrowth, surface roughness, IBAD

\section{Introduction}

Titanium has been widely used in bone implants, owing to its near-ideal mechanical strength and relative biocompatibility. However, these devices have limited capability in that they frequently necessitate revision surgery due to infection and poor osseointegration. It is estimated that up to $5 \%$ of all total knee and hip arthroplasties develop infection-related complications, which are one of the main reasons for revisions, second only to implant instability. ${ }^{1}$ Additionally, infection can exacerbate loosening at the bone-implant interface by causing an inflammatory response, preventing bone ingrowth and thus hastening implant failure. Implants can, however, be treated to encourage cell integration, and numerous coating processes have been tested as a way to improve bone integration and biological adaptivity of titanium. ${ }^{2-5}$ However, other than using systemic antibiotic delivery, there are few options clinically available to decrease the growing rate of infected orthopedic implants. Many groups have incorporated antibiotics directly into layered coatings on top of titanium surfaces, ${ }^{4,5}$ but the coating adhesion may not survive the wear that titanium implants can experience during handling, implantation and use. In the research community, the advent of nanoscale titanium texturing has resulted in improved osseointegration and decreased bacterial presence. ${ }^{6,7}$

Nanoscale features are commonly used in nature to achieve a number of novel effects without changing material composition. Insect wings, for example, employ
Correspondence: Thomas J Webster Department of Chemical Engineering, Northeastern University, 360 Huntington Ave, Boston, MA 02115, USA Email th.webster@neu.edu (c) (7) 2017 Stolzoff et al. This work is published and licensed by Dove Medical Press Limited. The full terms of this license are available at https://www.dovepress.com/terms.php
and incorporate the Creative Commons Attribution - Non Commercial (unported, v3.0) License (http://creativecommons.org/licenses/by-nc/3.0/). By accessing the work you hereby accept the Terms. Non-commercial uses of the work are permitted without any further permission from Dove Medical Press Limited, provided the work is properly attributed. For permission for commercial use of this work, please see paragraphs 4.2 and 5 of our Terms (https://www.dovepress.com/terms.php). 
arrays of nanoscale pillars on their wings to prevent biofouling and bacterial adhesion. ${ }^{8}$ The cicada wing, as an example, has highly uniform pillars with diameters ranging from 150 to $200 \mathrm{~nm}$ and spaced 9-48 nm apart, depending on the species of cicada. Generally, smaller and sharper pillars are more effective at preventing bacterial growth than larger ones. ${ }^{9}$ Pogodin et al ${ }^{10}$ have modeled the mechanism by which bacteria interact with these nanopillars previously, suggesting that bacteria will rupture upon attempts to adhere to these nanorough surfaces. ${ }^{11}$ Additionally, it is known that many biological tissues have nanoscale structures to provide the necessary scaffolds for healthy function, such as the strength and flexibility of bones due to the incorporation of nanocrystal bone minerals (single nm scale) in a matrix of $500 \mathrm{~nm}$ collagen fibers. ${ }^{12}$ For these reasons, nanofeatures have been incorporated into several engineered biomaterials to prevent bacterial colonization as well as to improve mammalian cell integration and reduce inflammatory responses to these materials.

It is now well established that nanoscale surface features incorporated into orthopedic implants improve bone growth and reduce bacterial adherence, but it is unknown exactly how the shape or structure of the texture affects bone growth and bacterial inhibition. ${ }^{13}$ While many treatments can create similar roughness measurements, such as adding coatings onto the surface via electrophoretic deposition, ${ }^{2}$ electrochemical anodizing $^{14-16}$ and silver nanoparticle-embedded hydrogelbased coatings, ${ }^{17}$ the morphology of the resulting surfaces can vary drastically. Lorenzetti et $\mathrm{al}^{11}$ used hydrothermal treatments to generate nanorough surfaces on titanium and found that macro- and microscale grooves (results of the initial material machining process) provided niches for bacteria to adhere and proliferate on, despite high roughness values. Silver is one of the most prominent non-small-molecule drugs used for antibacterial applications, ${ }^{3,17-19}$ but can be expensive and mildly toxic to surrounding mammalian cells. ${ }^{20,21}$ Additionally, adding different materials to the surface may lead to imperfectly fused layers that may eventually wear or delaminate from the bulk implant.

We hypothesize that in addition to being nanorough, it is important for implant surfaces, titanium in this case, to have specific geometries designed to efficiently prevent bacterial adhesion and growth while still promoting healthy osteoblast (bone-forming cell) growth and activity. Here, we employed ion beam evaporation to achieve highly adherent, fine-grained, pinhole-free coatings of exceptionally high quality without changing the bulk properties of the substrate material. Using this technique, we can control the roughness and topographical features of the titanium substrates to a very fine degree.

When creating nanorough surfaces, the typical goal has been to aim for specific roughness parameters to prevent bacterial adhesion, proliferation and biofilm formation. However, this approach may not be adequate. Rather than attempting to pinpoint only a precise roughness, we sought to find a connection between the roughness in combination with the topographical geometries and the degree of osteoblast growth observed on the corresponding surface. Additionally, we intended to create a more well-defined approach concerning how to quantify roughness, whether in terms of the quantity of finer nanoscale structures or in terms of the relative shape and size of the nanofeatures on the surfaces of the samples.

\section{Materials and methods Materials}

Unless otherwise noted, all chemicals and materials were purchased from Fisher Scientific.

\section{Titanium coupon preparation}

Test samples were treated with textured titanium films by our industry collaborator N2 Biomedical using ion beam-assisted deposition (IBAD). During the IBAD process, an electron beam evaporator is used to generate a vapor flux of atoms, which condenses on the substrate, while ions (typically Ar, $\mathrm{N}$ or $\mathrm{O}$ ) are simultaneously accelerated into the growing film at energies from hundreds to several thousand electron volts. This concurrent ion bombardment affects several film properties, including morphology, density, film stress, crystallinity and chemical composition. The porous, columnar microstructure that is observed in conventional physical vapor deposition film growth can be eliminated because energy from the ion beam causes increased nucleation density and increased surface mobility of the coating atoms. Through physical sputtering and redeposition, loosely bonded coating atoms are forced into voids, densifying the film as it grows. Additionally, high ion beam exposure breaks up crystal grains before they can grow to their natural size as described by the Thornton model. ${ }^{22}$ This can yield nanocrystalline or amorphous microstructure, with crystal size being a predictable function of the ion beam parameters.

All surfaces have some natural topography, and most have inclusions or grain defects resulting in small regions of differing density or crystal structure. These features will have different sputtering properties (sputter yield) than regions immediately adjacent to them. As a material is sputtered, 
either during evaporation or as an independent process, regions of high sputter yield will be preferentially removed, in effect causing lower sputter yield regions to be built up. In addition, faces of features normal to the ion beam are sputtered more, while the other, self-shielded faces are sputtered less. Over time, the imperceptible surface features become exaggerated, and a characteristic texture evolves. Control over these features can be achieved by selecting specific ion beam conditions and manipulating the exposure geometry. In this study, five different ion beam exposure conditions were used to produce titanium thin films of varying nanotexture.

The texturing of the samples described here was performed by applying IBAD films having a wide range of ion energy densities. The titanium coupons (Ti-6,4, McMaster Carr, grade 5) were polished to a mirror finish and coated with $0.5-1 \mu \mathrm{m}$ of textured titanium. To generate varied levels of nanotexture, the argon ion beam energy and current density were modified relative to the deposition rate. Five ion energy densities were examined, ranging from zero to as high as $90.3 \mathrm{~mJ} / \mathrm{cm}^{3}$ (Table 1).

\section{Sample sterilization}

For all cell studies, it was necessary to ensure that the tested materials were disinfected and sterilized to prevent any contamination and skewing of results. To sterilize the coupons in this study, they were first submerged in acetone and sonicated for 20 minutes. Acetone was removed inside a sterile laminar flow hood, and the samples were resubmerged in $70 \%$ ethanol, followed by another round of sonication. The ethanol was then removed in the sterile hood, and the samples were rinsed with phosphate buffered saline (PBS), dried and sterilized under ultraviolet overnight.

\section{X-ray photoelectron spectroscopy (XPS)}

To confirm that the treatment method used to achieve nanofeatured surfaces did not alter surface chemistry, XPS was performed on the two treated surfaces and compared to an untreated control, using a magnesium anode nonmonochromated X-ray source (Phi model 04-548) and a hemispherical analyzer (Phi model 10-360). Results were analyzed using a peak curve-fitting program (Multipack 9.3.0.3; Ulvac-Phi, Inc) while the background subtraction was performed using the integrated Shirley method.

\section{Atomic force microscopy (AFM)}

AFM was performed with a Parks Systems NX-10 microscope in noncontact mode to scan the sample surfaces, and XEI software was used to analyze the scans. A scan rate of $0.5 \mathrm{~Hz}$ at a set point between 12 and $15 \mathrm{~nm}$ was used with a Park Systems non-contact cantilever tip (PPP-NCHR). Scans were completed at $1 \times 1,2 \times 2$ and $5 \times 5 \mu \mathrm{m}$ sizes.

\section{Scanning electron microscopy (SEM)}

Samples were imaged using a Hitachi s-4800 SEM at $3 \mathrm{keV}$, magnified at $30 \mathrm{k}$. Images acquired were processed using ImageJ to measure feature sizes.

\section{Goniometry and surface energy}

A Phoenix 150 Contact Angle Measurement System (Kostas Center) was used to image and analyze static contact angle measurements of known solvents (water, glycerol, polyethylene glycol [200 $\mathrm{mW}$ ] and ethylene glycol) to calculate surface energy of the titanium coupons, with surface energy calculated using Young's equation. Small droplets of solvent $(\sim 20 \mu \mathrm{L})$ were applied to the titanium samples, and contact angle measurements were taken immediately, all at room temperature. Known surface tensions of liquids in Table 2 as described by Khang et $\mathrm{al}^{23}$ using the OwensWendt model (Equation 1) were used in the calculations for surface energy.

$$
1+\gamma_{1} \cos \theta=2\left(\sqrt{\gamma_{s}^{d} \gamma_{l}^{d}}+\sqrt{\gamma_{s}^{p} \gamma_{l}^{p}}\right)
$$

Table I Key manufacturing process differences to obtain the materials of interest to the present study

\begin{tabular}{|c|c|c|c|c|c|}
\hline \multirow{2}{*}{$\begin{array}{l}\text { Sample } \\
\text { type }\end{array}$} & \multirow{2}{*}{$\begin{array}{l}\text { Treatment } \\
\text { value }\left(\mathrm{mJ} / \mathrm{cm}^{3}\right)\end{array}$} & \multicolumn{2}{|c|}{ RMS roughness $(\mathrm{nm})$} & \multirow{2}{*}{$\begin{array}{l}\text { Water } \\
\text { contact angle }\end{array}$} & \multirow{2}{*}{$\begin{array}{l}\text { Surface } \\
\text { energy }\left(m J / m^{2}\right)\end{array}$} \\
\hline & & $2 \times 2 \mu \mathrm{m}$ & $5 \times 5 \mu \mathrm{m}$ & & \\
\hline Plain $\mathrm{Ti}$ & $\mathrm{N} / \mathrm{A}$ & $45.73 \pm 9.05$ & $66.33 \pm 0.23$ & $40.12 \pm 3.99$ & 60.09 \\
\hline Sample I & 90.3 & $29.60 \pm 0.14$ & $31.64 \pm 1.63$ & $42.03 \pm 4.09$ & 58.24 \\
\hline Sample 2 & 2.8 & $34.16 \pm 0.03$ & $38.50 \pm 0.02$ & $42.25 \pm 3.39$ & 55.24 \\
\hline Sample 3 & 15.3 & $30.02 \pm 0.03$ & $35.33 \pm 0.03$ & $44 \pm 5.50$ & 55.91 \\
\hline Sample 4 & 36.4 & $77.33 \pm 0.43$ & $|05.50 \pm 0.7|$ & $60.39 \pm 4.22$ & 43.02 \\
\hline Sample 5 & No ion beam & $36.36 \pm 0.02$ & $38.8 I \pm 0.06$ & $41.68 \pm 9.13$ & 56.37 \\
\hline
\end{tabular}

Notes: Data presented as mean \pm standard deviation. RMS roughness as measured by AFM at scans at $2 \times 2$ and $5 \times 5 \mu \mathrm{m}$. Unpolished (plain Ti) titanium had greater roughness values than all but the fourth sample. Water contact angle and measured surface energy show that sample 4 had the most hydrophobic and lowest surface energy of all samples (in bold, $P<0.05$ ).

Abbreviations: AFM, atomic force microscopy; N/A, not applicable; RMS, root mean squared. 
Table 2 Surface tension values used for each of the solvents

\begin{tabular}{llll}
\hline Substance & $\gamma_{l}^{d}\left(\mathrm{~mJ} / \mathrm{m}^{2}\right)$ & $\gamma_{l}^{p}\left(\mathrm{~mJ} / \mathrm{m}^{2}\right)$ & Total $\left(\mathrm{mJ} / \mathrm{m}^{2}\right)$ \\
\hline Water & 22.1 & 50.7 & 72.8 \\
Glycerol & 37 & 26.4 & 63.4 \\
Polyethylene glycol 200 & 30.8 & 12.1 & 42.8 \\
\hline
\end{tabular}

\section{Bacteria adhesion and proliferation}

A colony-forming unit (CFU) assay was performed to quantify the antibacterial properties of the nanotextured titanium samples. Staphylococcus aureus (ATCC, Strain 12600) was grown in 3\% tryptic soy broth (Sigma-Aldrich) and plated at a concentration of $10^{6}$ cells $/ \mathrm{mL}$ onto each titanium sample. Samples were incubated for 24 hours at $37^{\circ} \mathrm{C}$ in $5 \% \mathrm{CO}_{2}$, undisturbed. After incubation, samples were lightly rinsed to remove any nonadherent bacteria, transferred to conical tubes containing $5 \mathrm{~mL}$ of sterile PBS and vortexed for 1 minute to remove all bacteria from the surface. This bacterial solution was then diluted serially and plated on tryptic soy agar (Sigma-Aldrich) plates. These plates were incubated overnight, and colonies were counted to calculate the CFUs of bacteria that had grown onto the titanium samples.

\section{Mammalian cell culture}

Human osteoblasts (ATCC CRL-11372) in PromoCell Osteoblast Basal Medium were grown in osteoblast media (PromoCell) with 10\% Osteoblast Growth Medium Supplement Mix (PromoCell) and 1\% penicillin/streptomycin (Gibco) to evaluate mammalian cell responses to the nanorough surface treatments. Cells were seeded at 10,000 cells $/ \mathrm{mL}$ and grown for 7 days, with media replaced every other day. An MTS viability assay was performed to evaluate relative cell density and viability between samples as compared to control cells grown on tissue culture plates.

\section{Statistical analysis}

All experiments were conducted in triplicate and repeated three times each. Statistics were calculated using a Student's $t$-test, and significance was determined at $P<0.05$.

\section{Results}

\section{Topographical analysis}

3D image reconstructions in Figure 1 showed a drastic change in topographical landscapes according to sample treatment conditions. Nanotextured samples were found to have a range of nanoroughness as analyzed by AFM. Scans at $2 \times 2$ and $5 \times 5 \mu \mathrm{m}$ (Figure 1) showed a variety of topographies on each sample, with differences in peak height, diameter and spacing, as well as dual populations of large and small peaks (some in samples 1 and 3). Their associated roughness values (Table 1) demonstrate that a variety of topographies can have similar surface roughness (samples 2, 3 and 5). This suggests that achieving one roughness value for "optimal" biological response may not be the "end-all quality" desired for many kinds of biomaterials.

Peak density was calculated by the quantification of peaks via the line profiles of the 3D reconstructions (Figure 1, rightmost column). Peaks were defined as a point between two "troughs". While sample 4 had the greatest roughness, samples 1 and 2 had the greatest number of peaks per area (Figure 2) and were significantly higher $(P<0.05)$ than that of sample 4, while samples 1, 2 and 3 all had significantly more peaks/area than sample 5 .

Further visualization of the sample surfaces was achieved through scanning electron microscopy (Figure 3). As seen in the AFM scans, samples 1 and 2 were both fairly regular in their smaller, more rounded peaks, while samples 4 and 5 had larger, more jagged flake-like features. Sample 1 had small, oval, pebble-like peaks, and while sample 2 also had regularly spaced features, they were more faceted, often appearing as di- or trihedrals. In contrast, samples 3, 4 and 5 had varying sizes of flake-like features that, while very nanorough, were also very irregular. While sample 4 was the roughest of all samples, it also had larger valleys $(0.5-1 \mu \mathrm{m}$ as measured via ImageJ).

\section{Hydrophobicity and surface energy}

Similar to roughness, sample 4 had the largest water contact angle (Table 1) of all the samples, yet plain titanium did not follow suit. This suggests that the high degree of nanoscale roughness created on sample 4 added a degree of superhydrophobicity that can be obtained with nanorough surfaces. Surface energy calculations using the Owens-Wendt model reflected these changes in hydrophobicity, with more hydrophilic samples (such as plain titanium and sample 1) having higher surface energy values (60.09 and $58.24 \mathrm{~mJ} / \mathrm{m}^{2}$, respectively) as shown in Table 1.

\section{Elemental analysis}

Samples 1 and 4, having the greatest differences in surface roughness and topography, were compared to the untreated titanium surface using XPS analysis to ensure that no chemical changes occurred. Survey scans of untreated and IBAD-treated samples confirmed the presence of $\mathrm{Ti}$ and $\mathrm{O}$ as shown in Figure 4A. Trace amounts of silver and silicon were detected on the untreated side, which can be accounted for 

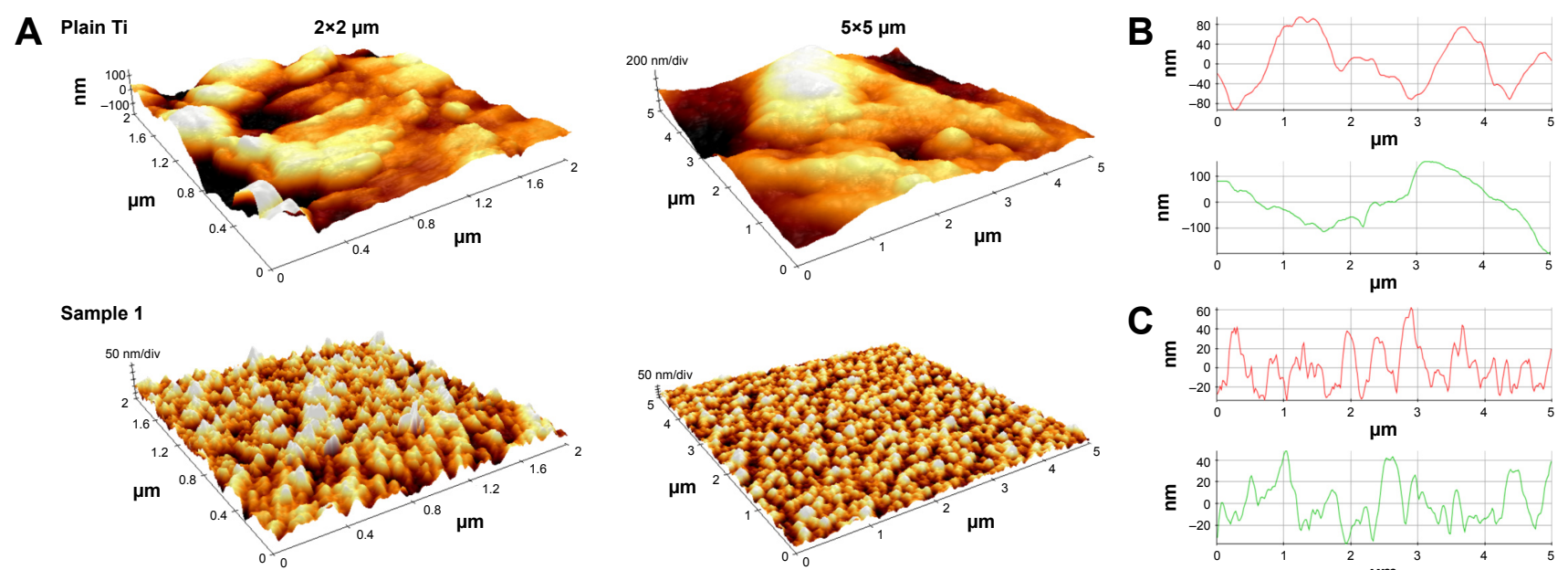

Sample 2
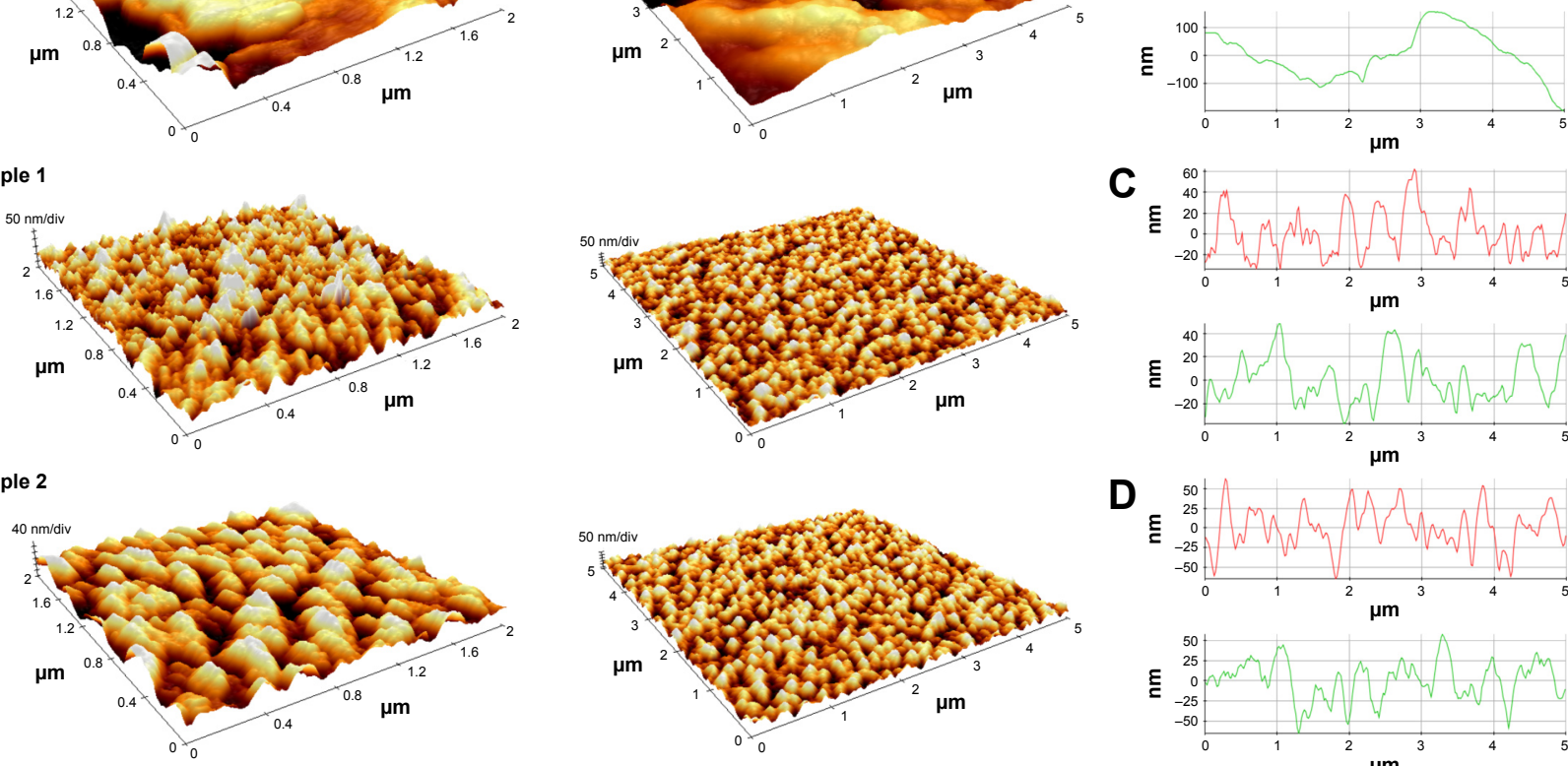

C.
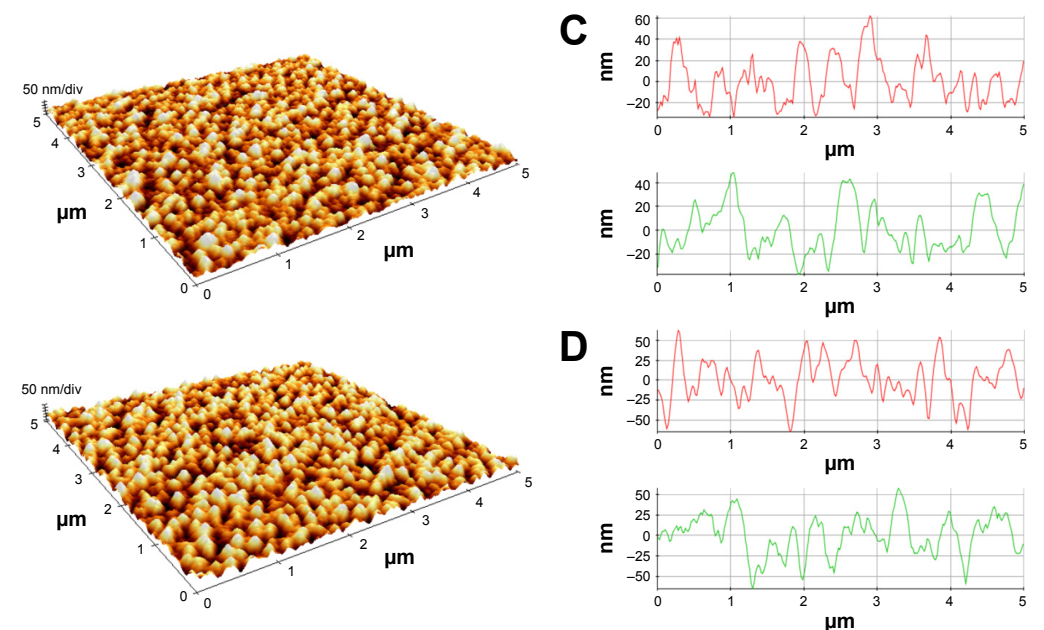

Sample 3
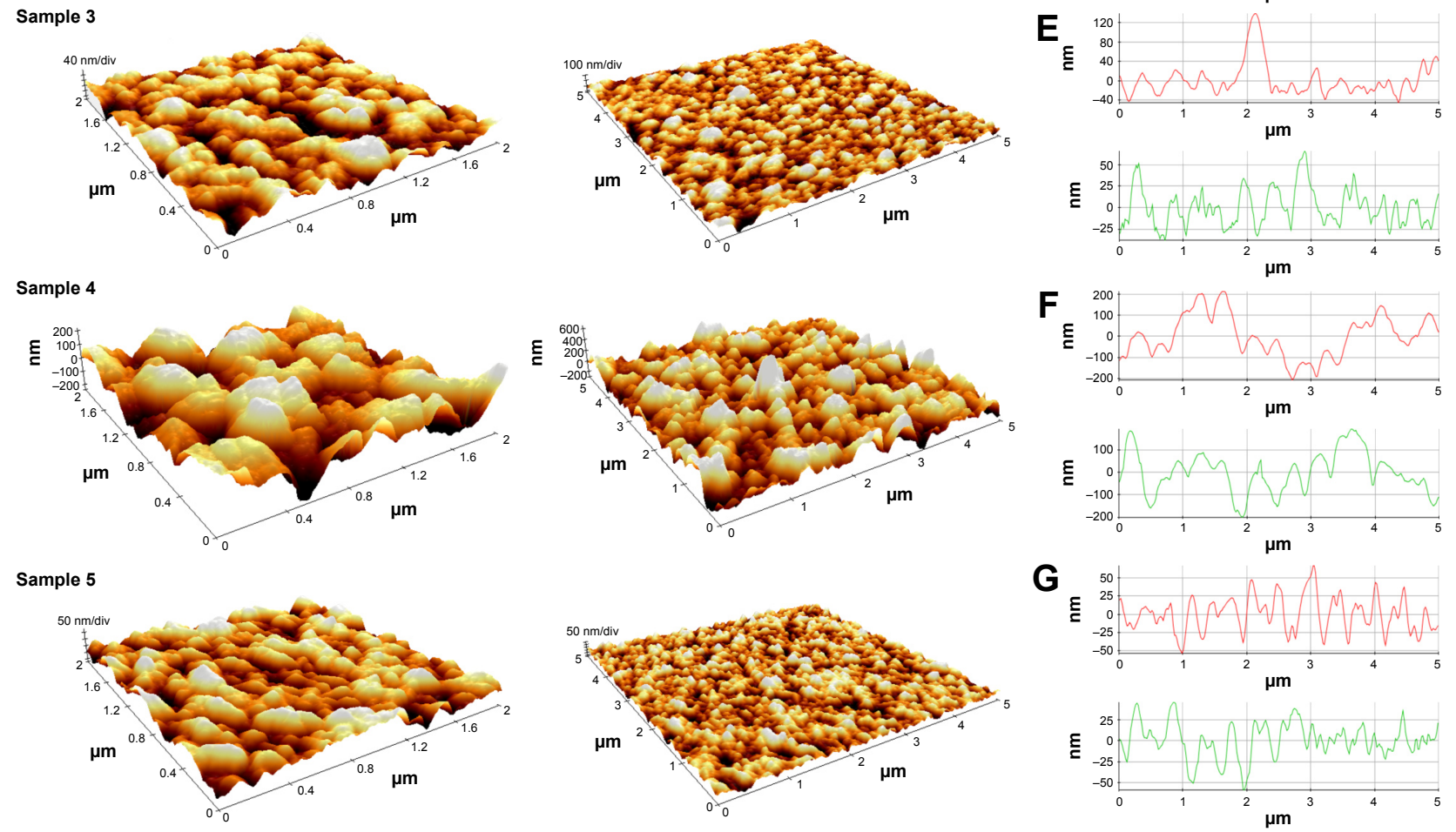

Figure I (A) Atomic force microscopy scans of plain titanium and samples I-5 (top to bottom) at $2 \times 2$ and $5 \times 5 \mu \mathrm{m}$ scans. Line profiles obtained for peak analysis (B-G) demonstrates the changes in peak density among the varied sample treatments.

by the use of those elements in other applications sharing the IBAD chamber. In addition, there were no significant changes in the bonding states of the Ti2p in either sample 1 or sample 4, and the atomic composition (Figure 4B) was consistent. This confirmed that the treatment method did not alter the surface chemistry despite drastic changes in surface topography.

\section{Bacterial growth}

Bacterial adhesion and proliferation, quantified by a CFU assay, demonstrated each sample's efficacy in reducing bacterial adhesion without the use of antibiotics as compared to untreated, unpolished titanium (Figure 5). Although sample 4 had the greatest roughness value of all samples, 


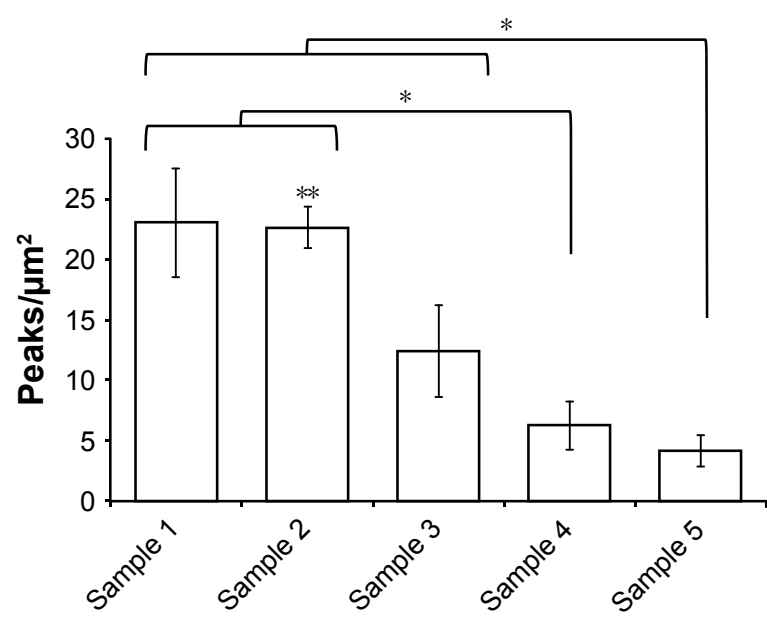

Figure 2 Peak density on each sample was calculated from AFM scans.

Notes: Samples $I$ and 2 had significantly greater $(P<0.05$ and $P<0.01)$ peak density than samples 4 and 5 , while sample 3 only had greater peak density than sample 5 $(P<0.05)$. $* P<0.05$, $* * P<0.01$. Data $=$ mean $\pm \mathrm{SEM}, \mathrm{N}=3$.

Abbreviations: AFM, atomic force microscopy; SEM, scanning electron microscopy.

it was samples 1 and 2 that showed the greatest reduction in bacterial growth, with sample 1 showing greater than a $1 \log$ decrease in bacterial density. Despite similar roughness values, sample 3 had the greatest amount of bacteria on the surface, further suggesting that both the roughness and topographical qualities must be considered in the development of antibacterial surface treatments. It is perhaps due to the intermittent large peaks that the bacteria could fit between and thus adhere to the surface. Several of these peaks were roughly $0.5-2 \mu \mathrm{m}$ apart in distance, which is enough for
S. aureus to fit between. Samples 4 and 5 similarly had the least number of peaks per area, but generally had more consistently sized peaks that may have prevented some of the excessive growth seen on sample 3 .

\section{Osteoblast proliferation}

Yet, even with this large difference in bacteria-material interactions, osteoblasts grown on these samples for 7 days (Figure 6) were generally found to be consistently more viable compared to control cells grown on polystyrene tissue culture plates. No significant difference $(P>0.05)$ was found between any of the treated titanium samples, though sample 2 seemed to have the greatest increase in osteoblast density of all the treated titanium coupons, showing a $\sim 150 \%$ increase in cell density compared to polystyrene controls.

\section{Discussion}

Samples 1 and 2 had similarly high peak density with similar dimensions ( $80 \mathrm{~nm}$ peak-to-base heights), but sample 2 possessed slightly more rounded peaks. As seen via SEM, both of these samples had smaller, more regularly shaped features than all other samples, despite having the highest and second-lowest ion beam density treatments as well as resulting film thickness. With a moderate increase in treatment intensity, sample 3's surface had a combination of smaller peaks $(50-80 \mathrm{~nm})$ and intermittent, large, jagged $(200 \mathrm{~nm})$ peaks, and with greater ion beam intensity used to generate sample 4, the sizes of these peaks intensified to as

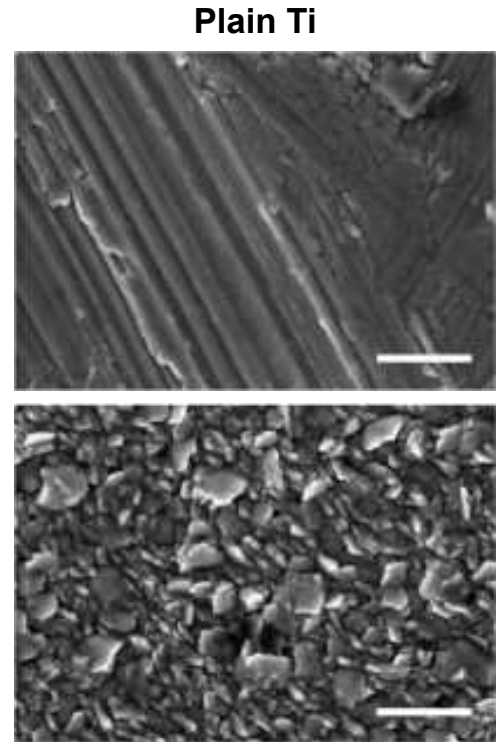

Sample 3

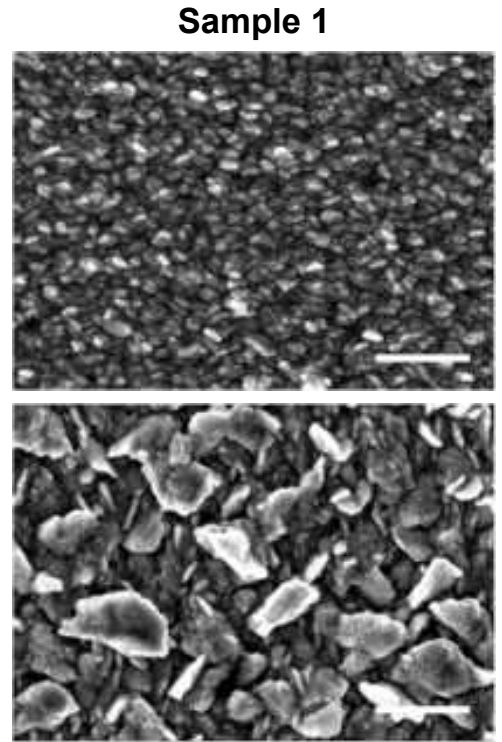

Sample 4

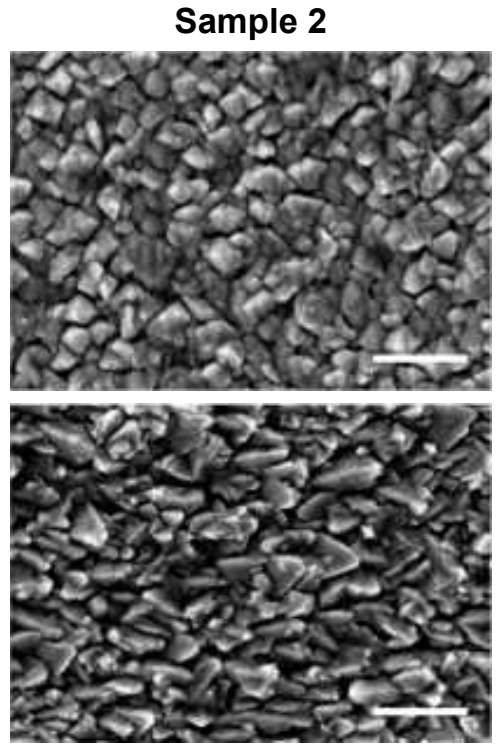

Sample 5

Figure 3 Scanning electron micrographs of the titanium samples show the drastic changes in topography despite mostly similar roughness measurements. Note: Scale bar $=500 \mathrm{~nm}$. 

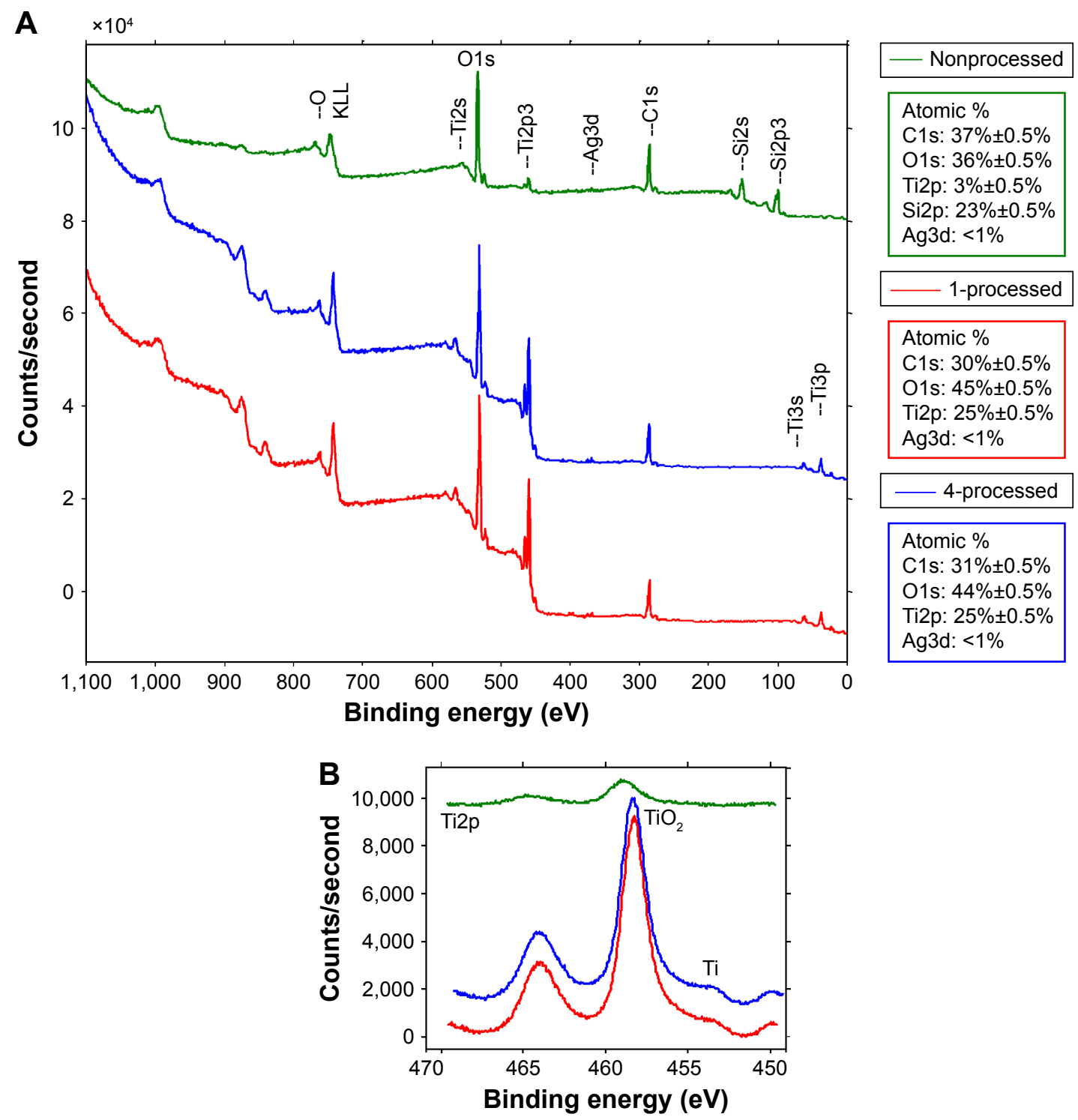

Figure 4 (A) XPS survey scan of samples I (red) and 4 (blue) compared to the untreated control, the underside of sample I (green). (B) XPS Ti2p tight scan, TiO ${ }_{2}$ peaks show no changes in composition of the treated samples.

Abbreviation: XPS, X-ray photoelectron spectroscopy.

high as $400 \mathrm{~nm}$. Sample 5 has similar peak heights $(80 \mathrm{~nm})$ but fewer in number per area, with wider, more rounded peaks than those of samples 1 and 2. It appears that with the midrange ion beam intensity, enough ions have been sputtered away that larger, jagged features are exposed, but as ion beam intensity increases, these features are eroded away until there is a regular pattern of smaller peaks.

Interestingly, despite these topographical changes, samples $1,2,3$ and 5 all had fairly similar surface roughness measurements but greatly differing bacterial response. As evidenced by the plain, untreated titanium's high roughness values and corresponding higher CFU count, "roughness" may not always equate to "antibacterial". Generally, the roughness values did not predict bacteria colony counts - if greater nanoroughness directly correlated to reduced bacterial adhesion, sample 4 would not have had the second highest CFU of the five treated samples. Perhaps unsurprisingly, it was samples 1 and 2, with the smaller, more closely packed peaks on the surface (similar to the nanopillars found on cicada wings) that had the fewest CFU of all the titanium samples. Regardless of nanoroughness, all titanium samples had greater osteoblast density than control polystyrene dishes.

These results demonstrate the need for further analysis of how implant surface morphology impacts both bacterial and human osteoblast interactions. With many of the commonly used instrumentation and characterization techniques used 


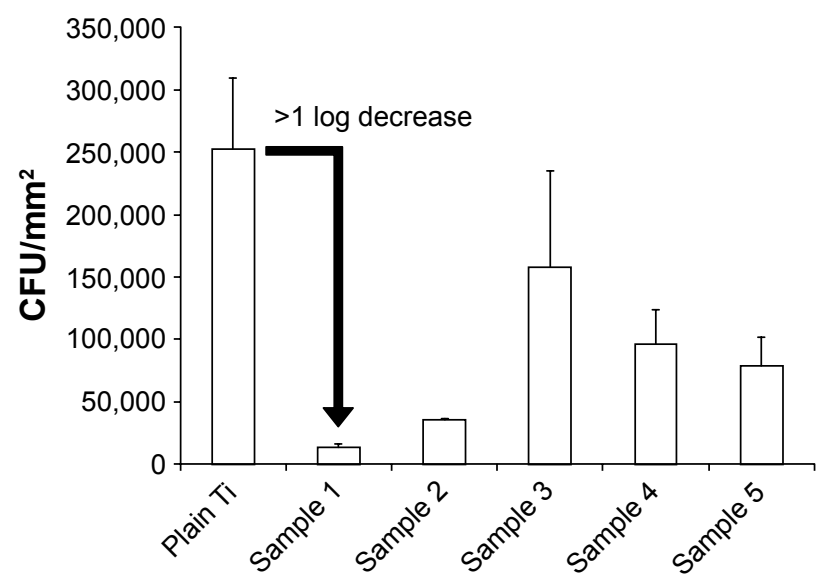

Figure 5 CFUs of Staphylococcus aureus on titanium samples after 24 hours of incubation.

Notes: Data $=$ mean \pm SEM, $N=3$.

Abbreviations: CFUs, colony forming units; SEM, scanning electron microscopy.

here and within literature, very similar values for roughness and surface energy can be achieved with very different surface features. By employing ion beam evaporation, we were able to generate five different titanium surface treatments that subtly changed the nanoscale topography and resulting cellular interactions without adding small molecule antibiotic drugs or growth factors.

\section{Conclusion}

Some nanorough surfaces have been established to be antibacterial, yet at the same time, beneficial to mammalian cell functions for improving medical implants. Additionally, it was believed that specific surface roughness values, in combination with surface energy measurements, were optimal for specific cellular interactions. Here, we established that several materials with similar roughness measurements, yet very

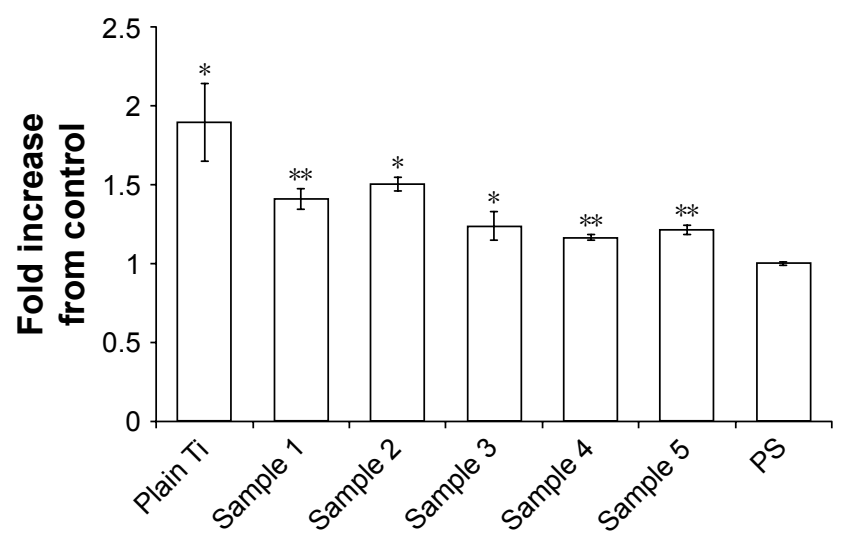

Figure 6 Osteoblast viability on titanium samples compared to PS controls after 7 days of incubation.

Notes: $* P<0.05,{ }^{*} * P<0.01$ compared to PS. Data $=$ mean $\pm S E M, N=3$.

Abbreviations: PS, polystyrene; SEM, scanning electron microscopy. different topographical qualities, can have significantly different cellular interactions without changing surface chemistry. Of the treated titanium coupons tested, samples 1 and 2 - despite having negligible differences in roughness compared to samples 3 and 5 - had the greatest decrease in bacterial adhesion, while sample 4 with the largest roughness value was no better. The main differences in samples 1 and 2 were that their topography included evenly spaced small peaks, compared to the larger, more randomly positioned peaks in samples 3 and 5 . It is evident that another, somewhat qualitative parameter - surface topography - is a key player in the prevention of bacterial adhesion and proliferation, not just the roughness and surface energy measurements. Thus, it is recommended to have a high density of consistently sized nanofeatures to best prevent bacterial adhesion while generating surfaces with nanoscale roughness, rather than simply aiming for high nanoroughness values.

\section{Acknowledgments}

The authors acknowledge financial support from the National Institutes of Health (NIH/NIDCR Grant\#1R43DE025526-01). The authors gratefully thank Robert Eagan and William Fowle for their technical assistance and the Northeastern University Department of Chemical Engineering for facilities and funding. Atomic force microscopy and contact angle measurements were performed in the George J. Kostas Nanoscale Technology and Manufacturing Research Center.

\section{Disclosure}

The authors report no conflicts of interest in this work.

\section{References}

1. Campoccia D, Montanaro L, Arciola CR. The significance of infection related to orthopedic devices and issues of antibiotic resistance. Biomaterials. 2006;27(11):2331-2339.

2. Mathew D, Bhardwaj G, Wang Q, et al. Decreased Staphylococcus aureus and increased osteoblast density on nanostructured electrophoreticdeposited hydroxyapatite on titanium without the use of pharmaceuticals. Int J Nanomedicine. 2014;9:1775.

3. Ewald A, Glückermann SK, Thull R, Gbureck U. Antimicrobial titanium/ silver PVD coatings on titanium. Biomed Eng Online. 2006;5(1):22.

4. Hizal F, Zhuk I, Sukhishvili S, Busscher HJ, van der Mei HC, Choi C-H. Impact of 3D hierarchical nanostructures on the antibacterial efficacy of a bacteria-triggered self-defensive antibiotic coating. ACS Appl Mater Interfaces. 2015;7(36):20304-20313.

5. Badar M, Rahim MI, Kieke M, et al. Controlled drug release from antibiotic-loaded layered double hydroxide coatings on porous titanium implants in a mouse model. J Biomed Mater Res Part A. 2015;103(6): 2141-2149.

6. Zhang W, Li Z, Liu Y, et al. Biofunctionalization of a titanium surface with a nano-sawtooth structure regulates the behavior of rat bone marrow mesenchymal stem cells. Int J Nanomedicine. 2012;7:4459.

7. Lu J, Webster TJ. Reduced immune cell responses on nano and submicron rough titanium. Acta Biomater. 2015;16:223-231. 
8. Hasan J, Webb HK, Truong VK, et al. Selective bactericidal activity of nanopatterned superhydrophobic cicada Psaltoda claripennis wing surfaces. Appl Microbiol Biotechnol. 2013;97(20):9257-9262.

9. Kelleher SM, Habimana O, Lawler J, et al. Cicada wing surface topography: an investigation into the bactericidal properties of nanostructural features. ACS Appl Mater Interfaces. 2016;8(24):14966-14974.

10. Pogodin S, Hasan J, Baulin VA, et al. Biophysical model of bacterial cell interactions with nanopatterned cicada wing surfaces. Biophys $J$. 2013;104(4):835-840.

11. Lorenzetti M, Dogša I, Stošicki T, et al. The influence of surface modification on bacterial adhesion to titanium-based substrates. ACS Appl Mater Interfaces. 2015;7(3):1644-1651.

12. Rho J-Y, Kuhn-Spearing L, Zioupos P. Mechanical properties and the hierarchical structure of bone. Med Eng Phys. 1998;20(2):92-102.

13. Puckett SD, Taylor E, Raimondo T, Webster TJ. The relationship between nanostructure of titanium surfaces and bacterial attachment. Biomaterials. 2010;31:706.

14. Yao C, Slamovich EB, Webster TJ. Enhanced osteoblast functions on anodized titanium with nanotube - like structures. J Biomed Mater Res Part A. 2008;85(1):157-166.

15. Kazemzadeh-Narbat M, Lai BFL, Ding C, Kizhakkedathu JN, Hancock REW, Wang R. Multilayered coating on titanium for controlled release of antimicrobial peptides for the prevention of implantassociated infections. Biomaterials. 2013;34(24):5969-5977.

16. Wang J, Li J, Qian S, et al. Antibacterial surface design of titaniumbased biomaterials for enhanced bacteria-killing and cell-assisting functions against periprosthetic joint infection. ACS Appl Mater Interfaces. 2016;8(17):11162-11178.
17. De Giglio E, Cafagna D, Cometa S, et al. An innovative, easily fabricated, silver nanoparticle-based titanium implant coating: development and analytical characterization. Anal Bioanal Chem. 2013; 405(2-3):805-816.

18. Lansdown ABG. A pharmacological and toxicological profile of silver as an antimicrobial agent in medical devices. Adv Pharmacol Sci. 2010; 2010:910686.

19. Song D-H, Uhm S-H, Lee S-B, Han J-G, Kim K-N. Antimicrobial silver-containing titanium oxide nanocomposite coatings by a reactive magnetron sputtering. Thin Solid Films. 2011;519(20):7079-7085.

20. Samberg ME, Oldenburg SJ, Monteiro-Riviere NA. Evaluation of silver nanoparticle toxicity in skin in vivo and keratinocytes in vitro. Environ Health Perspect. 2010;118(3):407.

21. Kim K-T, Truong L, Wehmas L, Tanguay RL. Silver nanoparticle toxicity in the embryonic zebrafish is governed by particle dispersion and ionic environment. Nanotechnology. 2013;24(11):115101.

22. Thornton JA. Structure-zone models of thin films. 31st Annual Technical Symposium. San Diego: International Society for Optics and Photonics; 1988:95-105.

23. Khang D, Kim SY, Liu-Snyder P, Palmore GTR, Durbin SM, Webster TJ. Enhanced fibronectin adsorption on carbon nanotube/poly(carbonate) urethane: independent role of surface nano-roughness and associated surface energy. Biomaterials. 2007;28(32):4756-4768.
International Journal of Nanomedicine

\section{Publish your work in this journal}

The International Journal of Nanomedicine is an international, peerreviewed journal focusing on the application of nanotechnology in diagnostics, therapeutics, and drug delivery systems throughout the biomedical field. This journal is indexed on PubMed Central, MedLine, CAS, SciSearch $\AA$, Current Contents $₫ /$ Clinical Medicine,

\section{Dovepress}

Journal Citation Reports/Science Edition, EMBase, Scopus and the Elsevier Bibliographic databases. The manuscript management system is completely online and includes a very quick and fair peer-review system, which is all easy to use. Visit http://www.dovepress.com/ testimonials.php to read real quotes from published authors. 